\title{
Safety of Ipragliflozin in Patients with Type 2 Diabetes Mellitus: Pooled Analysis of Phase II/III/IV Clinical Trials
}

\author{
Atsunori Kashiwagi - Marina V. Shestakova • Yuichiro Ito • \\ Masahiro Noguchi · Wim Wilpshaar · Satoshi Yoshida • \\ John P. H. Wilding
}

Received: August 12, 2019 / Published online: October 12, 2019

(C) The Author(s) 2019

\section{ABSTRACT}

Introduction: Ipragliflozin is a selective sodium-glucose cotransporter 2 (SGLT2) inhibitor approved for the treatment of type 2 diabetes mellitus (T2DM). The objective of this pooled analysis was to characterise the safety profile of ipragliflozin based on safety data from published randomised controlled trials.

Enhanced Digital Features To view enhanced digital features for this article go to https://doi.org/10.6084/ m9.figshare.9873650.

Electronic supplementary material The online version of this article (https://doi.org/10.1007/s13300019-00699-8) contains supplementary material, which is available to authorized users.

A. Kashiwagi ( $\square)$

Kusatsu General Hospital, Kusatsu, Shiga, Japan e-mail: kashiwagi@kusatsu-gh.or.jp

M. V. Shestakova

Endocrinology Research Centre, Diabetes Institute, Moscow, Russia

Y. Ito $\cdot$ M. Noguchi $\cdot$ S. Yoshida

Astellas Pharma Inc., Tokyo, Japan

W. Wilpshaar

Astellas Pharma Europe B.V., Leiden, The

Netherlands

J. P. H. Wilding

Obesity and Endocrinology Research, Institute of Ageing and Chronic Disease, University of Liverpool, Liverpool, UK
Methods: Safety data from 12 randomised, phase II/III/IV placebo-controlled, parallel group, comparative studies of ipragliflozin in patients with T2DM were pooled. Treatmentemergent adverse events (TEAEs) were analysed for patients who had received at least one dose of ipragliflozin $50 \mathrm{mg}(n=1209)$ or placebo ( $n=796)$ in studies lasting for up to 24 weeks. TEAEs of special interest and serious adverse events (SAEs) were assessed, as well as abnormal laboratory test and vital sign measurements.

Results: The overall incidences of TEAEs and SAEs between the ipragliflozin and placebo groups were similar, $63.8 \%$ vs $59.3 \%$ and $2.5 \%$ vs $3.3 \%$, respectively. The incidence of TEAEs leading to permanent discontinuation was lower for ipragliflozin (3.6\%) than placebo $(6.5 \%)$. The incidences of TEAEs of special interest including those related to urinary tract infection, cardiovascular events, renal disorder, fracture, malignant tumours and hypoglycaemia were also similar between the groups. Genital infections were more frequent with ipragliflozin $(2.4 \%)$ than placebo $(0.6 \%)$, as were pollakiuria/polyuria $(6.0 \%$ vs $2.0 \%)$, volume depletion $(4.9 \%$ vs $1.8 \%)$ and skin/subcutaneous tissue disorders ( $7.7 \%$ vs $4.4 \%)$. There were no reported cases of diabetic ketoacidosis, fractures, lower-limb amputation or Fournier's gangrene in ipragliflozin-treated patients across the 12 studies.

Conclusion: In randomised, placebo-controlled trials of patients with T2DM, ipragliflozin was 
well tolerated, with a similar overall incidence of TEAEs to placebo. No new safety signals were observed.

Trial Registration Numbers: NCT01071850, NCT00621868, NCT01057628, NCT01117584, NCT01135433, NCT01225081, NCT01242215, NCT02175784, NCT01505426, NCT02452632, NCT02794792, NCT01316094.

Funding: Astellas Pharma Inc.

Keywords: Ipragliflozin; Pooled analysis; Safety; Sodium-glucose cotransporter 2 inhibitor; Type 2 diabetes mellitus

\section{INTRODUCTION}

In patients with type 2 diabetes mellitus (T2DM), increased reabsorption of glucose from glomerular filtrate into the circulation is one of the key contributors to elevated blood glucose [1]. Glucose reabsorption is mediated primarily by sodium-glucose cotransporter 2 (SGLT2) of renal tubular cells, and patients with T2DM show enhanced levels of expression of the SGLT2 protein compared with healthy individuals [2].

Pharmacological SGLT2 inhibitors have been developed to lower blood glucose levels in T2DM by blocking its reabsorption and promoting urinary glucose excretion $[3,4]$. In addition to improved glycaemic control [5-16], SGLT2 inhibitors provide important clinical benefits for patients with T2DM. Unlike most other anti-hyperglycaemic agents, SGLT2 inhibitors promote weight loss [15, 17], lower blood pressure [18-21] and exert beneficial effects on other cardiometabolic risk factors, except for low-density lipoprotein cholesterol and nonhigh-density lipoprotein cholesterol [22]. Indeed, patients with T2DM at high risk for cardiovascular (CV) events have been shown to have a lower incidence of $\mathrm{CV}$ outcomes and death after treatment with SGLT2 inhibitors [23-27]. Furthermore, the insulin-independent mechanism means that the risk of hypoglycaemia is low and their action is not affected by progressive $\beta$-cell failure $[1,4]$. Thus, SGLT2 inhibitors can be used at any stage of T2DM management, and may also benefit patients with type 1 diabetes mellitus (T1DM) who have inadequate glycaemic control on insulin alone [28-31].

Overall, SGLT2 inhibitors are well-tolerated drugs, but some treatment-emergent adverse events (TEAEs) of special interest have been reported in clinical and post-marketing studies, including urinary tract infection (UTI), genital infection, volume depletion, ketoacidosis [32-34] and some cancers [32, 35, 36]. More recently, reports of increased risk of lower limb amputation [24], fractures [37], Fournier's gangrene [38] and skin and subcutaneous tissue disorders [39-42] have emerged.

Ipragliflozin is an SGLT2 inhibitor which is indicated for the treatment of T1DM, in combination with insulin, and T2DM, either as monotherapy or in combination with other anti-hyperglycaemic agents. It was the first SGLT2 inhibitor to be approved for T2DM in Japan (in 2014 [43]), and has since been approved in Korea and Russia. Individual studies and post-marketing surveillance data suggest that ipragliflozin is well tolerated [5-10, 18, 44-46]. However, safety data from single studies are limited by patient selection and population size, and surveillance data are limited by the lack of a comparator group. The current analysis aims to further establish the safety profile of ipragliflozin in patients with T2DM by analysing data from a large pool of comparative studies.

\section{METHODS}

\section{Study Design}

Data were pooled from 12 placebo-controlled, parallel group, comparative studies [5-16], of 12,16 or 24 weeks' duration (Table 1), in which adult patients with T2DM were randomised to treatment with ipragliflozin $50 \mathrm{mg}$ or placebo once daily. Seven of the studies were conducted in Japan, one in Korea, one in Korea and Taiwan, one in Russia and two were global studies (Table 1). In five studies, ipragliflozin treatment was extended open-label up to 52 weeks, while placebo treatment was administered up to a maximum of 24 weeks. As such, the 


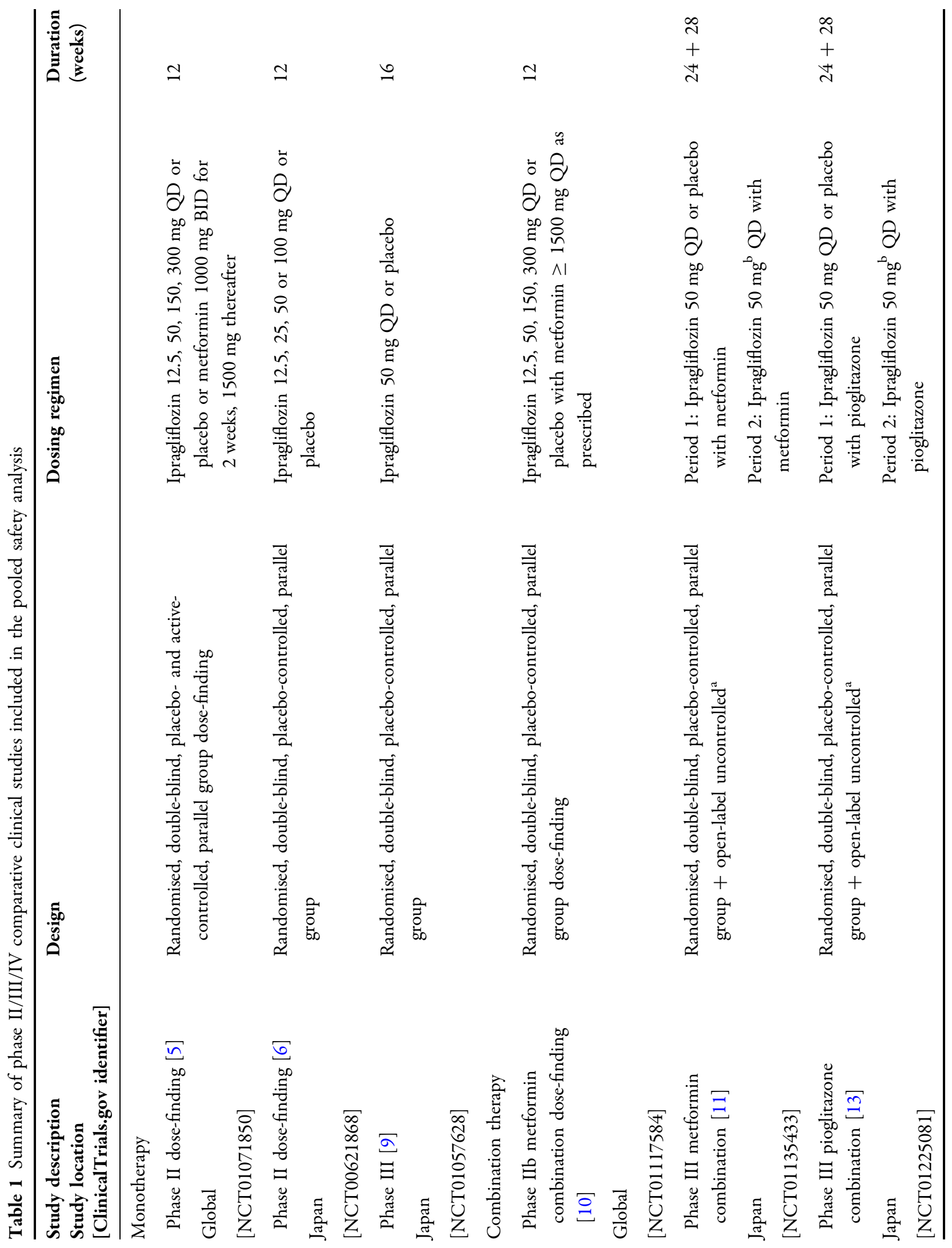




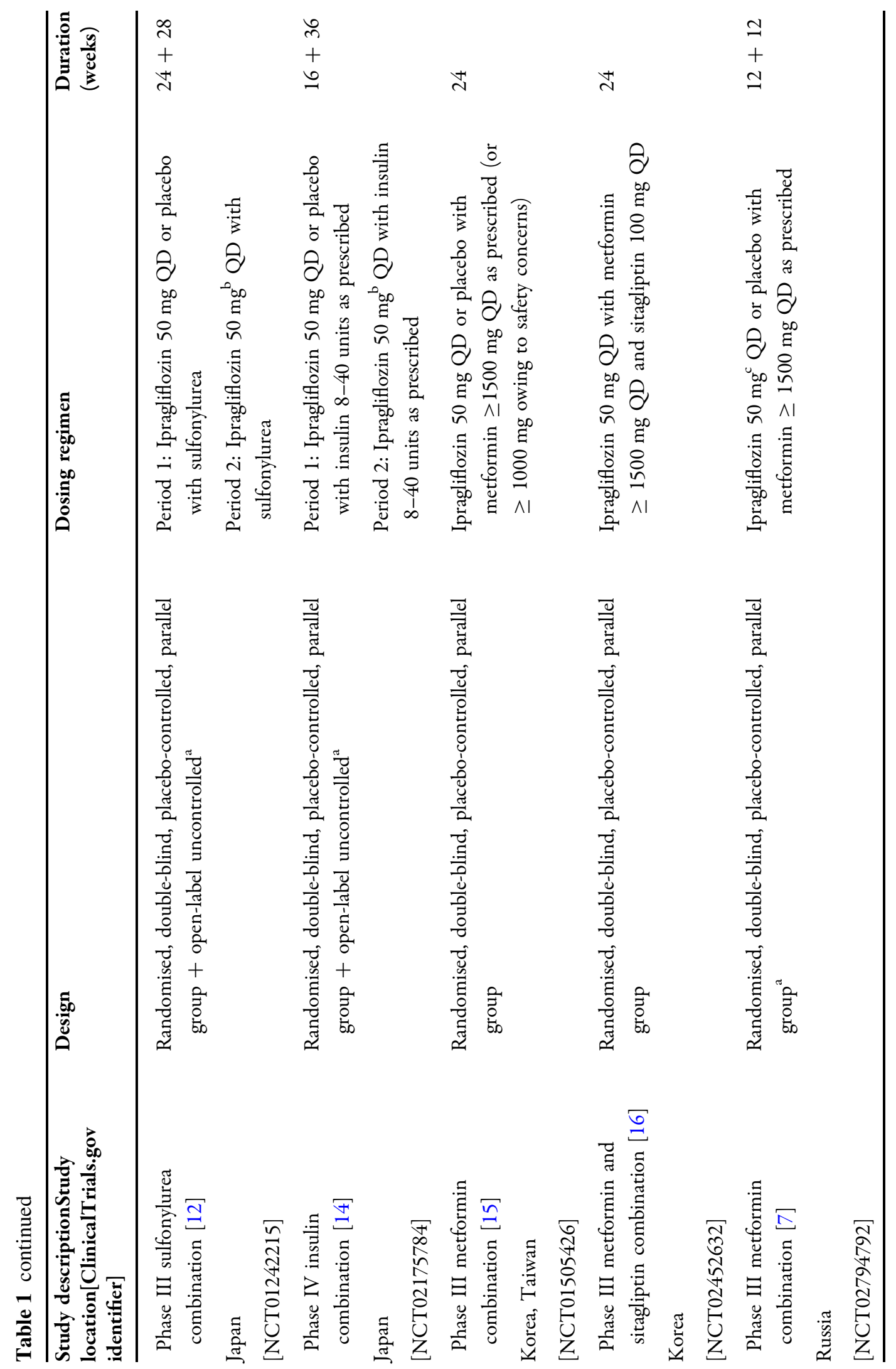




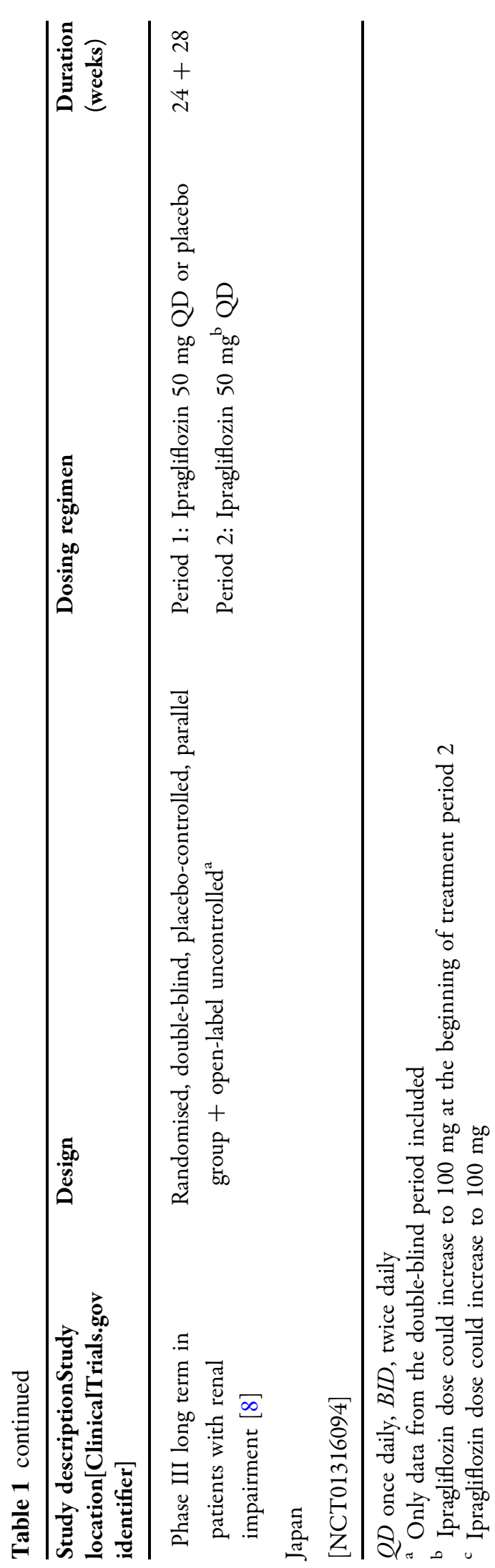

comparative pooled analysis only included data up to 24 weeks. While ipragliflozin is approved at $50 \mathrm{mg}$ and $100 \mathrm{mg}$ doses, only data for patients who received $50 \mathrm{mg}$ were included. Also, some phase II studies used doses that were not taken forward into clinical practice; these studies were excluded as they were not clinically relevant and might have distorted the results.

\section{Treatment Regimens}

Ipragliflozin was administered as monotherapy in three studies $[5,6,9]$, in combination with metformin in four studies [7, 10, 11, 15], as triple therapy with metformin and dipeptidyl peptidase 4 (DPP4) inhibitors in one study [16], and in combination with pioglitazone [13], sulfonylurea [12] and insulin [14] in one study each (Table 1). In a further study of patients with renal impairment, ipragliflozin was added to existing therapy, which may have consisted of diet and exercise alone, or in combination with an $\alpha$-glucosidase inhibitor, sulfonylurea or pioglitazone [8].

\section{Ethics}

This article is based on analysis of data from previously conducted studies. All procedures followed in the original studies involving human participants were in accordance with the ethical standards of the institutional and/or national research committee, and with the 1964 Helsinki declaration and its later amendments or comparable ethical standards. Informed consent was obtained from all individual participants included in the study. An independent ethics committee or institutional review board approved the clinical protocol at each participating centre. All participants provided written informed consent prior to inclusion.

\section{Outcomes}

TEAEs were analysed for the pooled safety analysis set, consisting of all patients who took at least one dose of ipragliflozin. TEAEs were defined as adverse events (AEs) that occurred after the initiation of study medication. The 
definition of TEAEs applied in each individual study was used for the analysis. TEAEs were summarised as follows: overall incidence of TEAEs, number of TEAEs, incidence of drugrelated TEAEs, TEAEs leading to permanent discontinuation, serious AEs (SAEs) and TEAEs occurring in at least $2 \%$ of the pooled population. A drug-related TEAE was defined as any TEAE with at least a possible relationship to the study treatment as assessed by the investigator, or with missing assessment of the causal relationship.

TEAEs were coded according to preferred terms in the Medical Dictionary for Regulatory Activities [MedDRA] version 19.1 and events recorded in studies using earlier versions of MedDRA were recoded. TEAEs were categorised by the investigators as mild, moderate or severe. Safety outcomes of special interest (hypoglycaemia, renal disorder, volume depletion, UTI, genital infection, pollakiuria or polyuria, $\mathrm{CV}$ events, fracture, malignant tumour, skin and subcutaneous tissue disorders) were identified using data captured in the case report form and a list of MedDRA search terms to programmatically identify AEs of interest (see Supplementary Table S1). A medical doctor reviewed all TEAEs, without any treatment information, and judged the relationship to AEs of special interest.

Descriptive subgroup analyses were performed for TEAEs according to ipragliflozin use alone or in combination with other glucoselowering drug regimens, including metformin, pioglitazone, $\alpha$-glucosidase inhibitor, sulfonylurea, DPP4 inhibitor, insulin, insulin and DPP4 inhibitor, and metformin and DPP4 inhibitor.

Abnormal laboratory findings (e.g. laboratory parameters, vital signs, electrocardiograms, physical findings) meeting the following criteria were considered AEs: causes clinical signs or symptoms; requires aggressive treatment; requires interruption or discontinuation of the study drug; and physical or laboratory findings considered by the investigator as clinically significant. The levels of ketone bodies were also assessed on the basis of laboratory results.

\section{Statistical Analysis}

Descriptive statistics were used to describe safety parameters. All patients who received at least one dose of study treatment were included in the analyses. The analyses were post hoc and no statistical hypothesis testing was performed. All data analyses were performed using SAS Version 9.3.

\section{RESULTS}

\section{Patient Demographics}

A total of 1209 ipragliflozin (50 mg)-treated patients and 796 placebo-treated patients were included in the pooled analysis. Baseline demographics and clinical characteristics were similar between the two treatment groups (Table 2). The majority of patients were male $(59.4 \%)$, had a mean age of around 58 years, were overweight (mean body mass index $27.2 \mathrm{~kg} / \mathrm{m}^{2}$ ) and had a mean duration of T2DM of approximately 8 years.

\section{Summary of Adverse Events}

The incidence of TEAEs (Table 3) was similar in patients treated with ipragliflozin (63.8\%) and placebo (59.3\%). Most TEAEs with ipragliflozin were mild in severity (89.0\%), and the incidence of SAEs was very low and similar for ipragliflozin $(2.5 \%)$ and placebo (3.3\%). No SAE occurred in more than two patients. The incidence of TEAEs leading to permanent discontinuation was lower for ipragliflozin (3.6\%) than placebo $(6.5 \%)$. Of the TEAEs that occurred at an incidence of at least $2 \%$ (Table 4 ), the incidence of constipation, pollakiuria and thirst were 2-3.6fold higher in the ipragliflozin group compared with placebo.

The overall TEAE incidences in patients treated with other doses of ipragliflozin in the pooled comparative trials are shown in Supplementary Table S2. There were no notable differences in the incidence of TEAEs, SAEs or TEAEs leading to permanent discontinuation 
Table 2 Patient baseline characteristics of patients treated with ipragliflozin $50 \mathrm{mg}$ or placebo (pooled comparative studies)

\begin{tabular}{|c|c|c|}
\hline Patient characteristics & $\begin{array}{l}\text { Ipragliflozin } 50 \mathrm{mg} \\
(N=1209)\end{array}$ & $\begin{array}{l}\text { Placebo } \\
(N=796)\end{array}$ \\
\hline \multicolumn{3}{|l|}{ Sex, $n(\%)$} \\
\hline Female & $475(39.3)$ & $339(42.6)$ \\
\hline Male & $734(60.7)$ & $457(57.4)$ \\
\hline \multicolumn{3}{|l|}{ Race, $n(\%)$} \\
\hline White & $209(17.3)$ & $151(19.0)$ \\
\hline Black & $9(0.7)$ & $11(1.4)$ \\
\hline Asian & $979(81.0)$ & $618(77.6)$ \\
\hline Other & $12(1.0)$ & $16(2.0)$ \\
\hline Age (years) & $58.1(10.3)$ & $57.4(9.9)$ \\
\hline Height $(\mathrm{cm})$ & $163.9(9.2)$ & $163.4(8.9)$ \\
\hline Weight $(\mathrm{kg})$ & $73.4(16.1)$ & $72.9(15.4)$ \\
\hline BMI $\left(\mathrm{kg} / \mathrm{m}^{2}\right)$ & $27.2(4.8)$ & $27.2(4.8)$ \\
\hline \multicolumn{3}{|l|}{ BMI, $n(\%)$} \\
\hline$<25 \mathrm{~kg} / \mathrm{m}^{2}$ & $456(37.7)$ & $300(37.7)$ \\
\hline$\geq 25 \mathrm{~kg} / \mathrm{m}^{2}$ & $753(62.3)$ & $496(62.3)$ \\
\hline Disease duration (months) ${ }^{a}$ & $102.5(81.9)$ & $97.5(79.1)$ \\
\hline $\mathrm{HbA}_{1 \mathrm{c}}(\%)^{\mathrm{b}}$ & $8.19(0.81)$ & $8.14(0.82)$ \\
\hline $\mathrm{eGFR}\left(\mathrm{mL} / \mathrm{min} / 1.73 \mathrm{~m}^{2}\right)^{\mathrm{b}}$ & $88.8(29.2)$ & $93.5(35.6)$ \\
\hline \multicolumn{3}{|l|}{ eGFR, $n(\%)^{\mathrm{b}}$} \\
\hline$>90 \mathrm{~mL} / \mathrm{min} / 1.73 \mathrm{~m}^{2}$ & $483(40.0)$ & $360(45.2)$ \\
\hline$\geq 60-<90 \mathrm{~mL} / \mathrm{min} / 1.73 \mathrm{~m}^{2}$ & $627(51.9)$ & $383(48.1)$ \\
\hline$\geq 30-<60 \mathrm{~mL} / \mathrm{min} / 1.73 \mathrm{~m}^{2}$ & $96(8.0)$ & $53(6.7)$ \\
\hline$<30 \mathrm{~mL} / \mathrm{min} / 1.73 \mathrm{~m}^{2}$ & $1(0.1)$ & $0(0)$ \\
\hline
\end{tabular}

Data are mean $(\mathrm{SD})$, unless otherwise indicated, in participants who received at least one dose of study drug $B M I$ body mass index, $H b A_{1 c}$ glycated haemoglobin Alc, $e G F R$ estimated glomerular filtration rate by Modification of Diet in Renal Disease (MDRD) equation

${ }^{a}$ Ipragliflozin, $n=1201$; placebo, $n=790$

b Ipragliflozin, $n=1207$; placebo, $n=796$ 
Table 3 Overview of adverse events in the pooled ipragliflozin comparative studies

\begin{tabular}{lcc}
\hline & $\begin{array}{l}\text { Ipragliflozin } \\
\boldsymbol{n}(\mathbf{5 0} \mathbf{~ \mathbf { ~ g }}(\boldsymbol{N}=\mathbf{1 2 0 9})\end{array}$ & $\begin{array}{l}\text { Placebo }(\boldsymbol{N}=\mathbf{7 9 6}) \\
\boldsymbol{n}(\%)\end{array}$ \\
\hline TEAE & $771(63.8)$ & $472(59.3)$ \\
Drug-related TEAE & $299(24.7)$ & $135(17.0)$ \\
SAE & $30(2.5)$ & $26(3.3)$ \\
Drug-related SAE & $5(0.4)$ & $6(0.8)$ \\
TEAE leading to discontinuation & $44(3.6)$ & $52(6.5)$ \\
Drug-related TEAE leading to discontinuation & $26(2.2)$ & $14(1.8)$ \\
TEAE severity & & \\
Mild & $686(56.7)$ & $397(49.9)$ \\
Moderate & $79(6.5)$ & $65(8.2)$ \\
Severe & $6(0.5)$ & $10(1.3)$ \\
\hline
\end{tabular}

$S A E$ serious adverse event, TEAE treatment-emergent adverse event

${ }^{a}$ Patient counted once under maximum severity

Table 4 Summary of treatment-emergent adverse events that occurred with ipragliflozin treatment at a frequency of at least 2\% (by MedDRA preferred term) in the pooled ipragliflozin comparative studies (excluding hypoglycaemia ${ }^{a}$ )

\begin{tabular}{llc}
\hline Adverse event characteristics & $\begin{array}{l}\text { Ipragliflozin } \\
\boldsymbol{n}(\%)\end{array}$ & $\begin{array}{l}\text { Placebo } \mathbf{~ m g}(\boldsymbol{N}=\mathbf{1 2 0 9}) \\
\boldsymbol{n}(\%)\end{array}$ \\
\hline Nasopharyngitis & $199(16.5)$ & $117(14.7)$ \\
Pollakiuria & $65(5.4)$ & $12(1.5)$ \\
Constipation & $37(3.1)$ & $12(1.5)$ \\
Thirst & $31(2.6)$ & $6(0.8)$ \\
\hline
\end{tabular}

Participants treated with at least one dose of study drug

MedDRA Medical Dictionary for Regulatory Activities

${ }^{a}$ Hypoglycaemia events are excluded from this summary owing to the effects of other glucose-lowering medications

compared with placebo or with ascending ipragliflozin doses.

\section{Adverse Events of Special Interest}

TEAEs related to pollakiuria, volume depletion, genital infection, and skin and subcutaneous tissue disorders were more frequent with ipragliflozin than with placebo (Table 5; see Supplementary Table S1 for AE definition criteria).

Stratification by glucose-lowering medication showed that hypoglycaemia was only increased when ipragliflozin was combined with insulin, owing to the hypoglycaemic effects of insulin (see "Hypoglycaemia-Related Events"), and therefore hypoglycaemia was not considered as a TEAE with increased incidence upon ipragliflozin treatment.

Events consistent with genital infection were experienced by 29 (2.4\%) patients in the ipragliflozin group and $5(0.6 \%)$ patients in the placebo group; vulvovaginal candidiasis and genital pruritus were the most commonly reported in the ipragliflozin group (see Supplementary Table S3). Pollakiuria/polyuria-related events occurred in $6.0 \%$ of ipragliflozin-treated 
Table 5 Incidence of treatment-emergent adverse events of special interest for ipragliflozin $(50 \mathrm{mg})$ in the pooled comparative studies

\begin{tabular}{|c|c|c|c|c|c|c|c|}
\hline \multirow[t]{2}{*}{$N(\%)$} & \multirow[t]{2}{*}{ TEAE } & \multirow{2}{*}{$\begin{array}{l}\text { Drug- } \\
\text { related } \\
\text { TEAE }\end{array}$} & \multirow{2}{*}{$\begin{array}{l}\text { TEAE } \\
\text { leading to } \\
\text { discontinuation }\end{array}$} & \multirow{2}{*}{$\begin{array}{l}\text { Drug-related TEAE } \\
\text { leading to } \\
\text { discontinuation }\end{array}$} & \multicolumn{3}{|c|}{ TEAE severity $^{\mathrm{a}}$} \\
\hline & & & & & Mild & Moderate & Severe \\
\hline \multicolumn{8}{|c|}{ Urinary tract infection } \\
\hline Ipragliflozin & $37(3.1)$ & $27(2.2)$ & $3(0.2)$ & $3(0.2)$ & $4(0.3)$ & $33(2.7)$ & 0 \\
\hline Placebo & $27(3.4)$ & $15(1.9)$ & 0 & 0 & $24(3.0)$ & $3(0.4)$ & 0 \\
\hline \multicolumn{8}{|c|}{ Genital infection } \\
\hline Ipragliflozin & $29(2.4)$ & $22(1.8)$ & $1(0.1)$ & $1(0.1)$ & $24(2.0)$ & $4(0.3)$ & $1(0.1)$ \\
\hline Placebo & $5(0.6)$ & $3(0.4)$ & $1(0.1)$ & $1(0.1)$ & $5(0.6)$ & 0 & 0 \\
\hline \multicolumn{8}{|c|}{ Pollakiuria or polyuria } \\
\hline Ipragliflozin & $72(6.0)$ & $65(5.4)$ & $1(0.1)$ & $1(0.1)$ & $70(5.8)$ & $2(0.2)$ & 0 \\
\hline Placebo & $16(2.0)$ & $12(1.5)$ & 0 & 0 & $16(2.0)$ & 0 & 0 \\
\hline \multicolumn{8}{|c|}{ Volume depletion } \\
\hline Ipragliflozin & $59(4.9)$ & $36(3.0)$ & $2(0.2)$ & $2(0.2)$ & $57(4.7)$ & $2(0.2)$ & 0 \\
\hline Placebo & $14(1.8)$ & $5(0.6)$ & $1(0.1)$ & 0 & $12(1.5)$ & $2(0.3)$ & 0 \\
\hline \multicolumn{8}{|c|}{ Cardiovascular events } \\
\hline Ipragliflozin & $1(0.1)$ & 0 & $1(0.1)$ & 0 & 0 & $1(0.1)$ & 0 \\
\hline Placebo & $4(0.5)$ & $3(0.4)$ & $3(0.4)$ & $3(0.4)$ & $1(0.1)$ & 0 & $3(0.4)$ \\
\hline \multicolumn{8}{|l|}{ Fracture } \\
\hline Ipragliflozin & $7(0.6)$ & 0 & $1(0.1)$ & 0 & $4(0.3)$ & $2(0.2)$ & $1(0.1)$ \\
\hline Placebo & $5(0.6)$ & 0 & 0 & 0 & $2(0.3)$ & $3(0.4)$ & 0 \\
\hline \multicolumn{8}{|l|}{ Renal disorder } \\
\hline Ipragliflozin & $11(0.9)$ & $3(0.2)$ & $1(0.1)$ & 0 & $9(0.7)$ & $1(0.1)$ & $1(0.1)$ \\
\hline Placebo & $13(1.6)$ & $5(0.6)$ & $1(0.1)$ & 0 & $13(1.6)$ & 0 & 0 \\
\hline \multicolumn{8}{|c|}{ Malignant tumours } \\
\hline Ipragliflozin & $3(0.2)$ & 0 & $2(0.2)$ & 0 & $2(0.2)$ & $1(0.1)$ & 0 \\
\hline Placebo & $3(0.4)$ & $1(0.1)$ & $2(0.3)$ & $1(0.1)$ & $1(0.1)$ & $1(0.1)$ & $1(0.1)$ \\
\hline \multicolumn{8}{|c|}{ Skin and subcutaneous tissue disorders } \\
\hline Ipragliflozin & $93(7.7)$ & $31(2.6)$ & $12(1.0)$ & $11(0.9)$ & $85(7.0)$ & $6(0.5)$ & $2(0.2)$ \\
\hline Placebo & $35(4.4)$ & $7(0.9)$ & $1(0.1)$ & 0 & $33(4.1)$ & $2(0.3)$ & 0 \\
\hline
\end{tabular}

For definitions for adverse events of special interest, see Supplementary Table S1

TEAE treatment-emergent adverse event

${ }^{a}$ Patient counted once under maximum severity 
Table 6 Change from baseline in urine ketone levels in the pooled comparative ipragliflozin studies

\begin{tabular}{|c|c|c|c|c|}
\hline \multirow[t]{2}{*}{ Time point } & \multicolumn{2}{|c|}{ Ipragliflozin $50 \mathrm{mg}(N=1209)$} & \multicolumn{2}{|c|}{ Placebo $(N=796)$} \\
\hline & $\overline{1+n(\%)^{\mathrm{a}}}$ & $3+n(\%)^{\mathbf{b}}$ & $1+n(\%)^{\mathrm{a}}$ & $3+n(\%)^{\mathrm{b}}$ \\
\hline Baseline & $22 / 1206(1.8)$ & $4 / 1206(0.3)$ & $15 / 795(1.9)$ & $1 / 795(0.1)$ \\
\hline Week 2 & $72 / 945(7.6)$ & $2 / 945(0.2)$ & $11 / 600(1.8)$ & 0 \\
\hline Week 12 & $61 / 1140(5.4)$ & $7 / 1140(0.6)$ & $12 / 702(1.7)$ & 0 \\
\hline End of treatment period & $58 / 1202(4.8)$ & $6 / 1202(0.5)$ & $9 / 792(1.1)$ & 0 \\
\hline
\end{tabular}

a Number of patients with $1+$ or higher $(2+, 3+$ or $4+)$ /number of patients with available laboratory values at each time point $(\%)$

b Number of patients with $3+$ or $4+/$ number of patients with available laboratory values at each time point (\%)

Table 7 Incidence of treatment-emergent adverse events related to hypoglycaemia in the pooled comparative ipragliflozin (50 mg) studies stratified by combination with insulin

\begin{tabular}{|c|c|c|c|c|c|c|c|c|}
\hline & \multicolumn{2}{|c|}{ No combination } & \multicolumn{2}{|c|}{ Insulin combinations } & \multicolumn{2}{|l|}{$\begin{array}{l}\text { Non-insulin } \\
\text { combinations }\end{array}$} & \multicolumn{2}{|l|}{ Overall } \\
\hline & $\begin{array}{l}\text { Ipragliflozin } \\
50 \mathrm{mg}\end{array}$ & Placebo & $\begin{array}{l}\text { Ipragliflozin } \\
50 \mathrm{mg}\end{array}$ & Placebo & $\begin{array}{l}\text { Ipragliflozin } \\
50 \mathrm{mg}\end{array}$ & Placebo & $\begin{array}{l}\text { Ipragliflozin } \\
50 \mathrm{mg}\end{array}$ & Placebo \\
\hline $\begin{array}{l}\text { Hypoglycaemia, } \\
n / N(\%)\end{array}$ & $\begin{array}{r}3 / 237 \\
(1.3)\end{array}$ & $\begin{array}{l}2 / 215 \\
(0.9)\end{array}$ & $\begin{array}{r}52 / 175 \\
(29.7)\end{array}$ & $\begin{array}{l}13 / 87 \\
(14.9)\end{array}$ & $\begin{array}{r}21 / 797 \\
(2.6)\end{array}$ & $\begin{array}{r}10 / 494 \\
(2.0)\end{array}$ & $\begin{array}{c}76 / 1209 \\
(6.3)\end{array}$ & $\begin{array}{c}25 / 796 \\
(3.1)\end{array}$ \\
\hline
\end{tabular}

patients compared with $2.0 \%$ of patients in the placebo group (Table 5). Events related to skin and subcutaneous tissue disorders occurred in $7.7 \%$ of ipragliflozin-treated patients compared with $4.4 \%$ of patients in the placebo group, and were mostly mild in severity (Table 5).

There was a similar incidence of TEAEs related to urinary tract infection in the ipragliflozin and placebo groups $(3.1 \%$ and $3.4 \%$, respectively, Table 5). The incidence of TEAEs related to CV events was low and similar between the two groups (Table 5; hazard ratio vs placebo $0.28,95 \%$ confidence interval [CI] 0.05-1.57). Four events occurred in the placebo group (acute myocardial infarction, cerebral infarction, thalamic infarction and left ventricular failure) and one event in the group treated with ipragliflozin $50 \mathrm{mg}$ (cerebral infarction).

The proportion of patients who had urine ketone body levels of at least $1+$ or $3+$ at the end of the treatment period was greater in the ipragliflozin group $(4.8 \%$ and $0.5 \%$, respectively) than in the placebo group $(1.1 \%$ and $0 \%$, respectively; Table 6). However, the number of subjects with $3+$ ketone levels was small and no TEAE related to ketoacidosis occurred.

\section{Hypoglycaemia-Related Events}

Overall, hypoglycaemia-related AEs were more frequent with ipragliflozin than with placebo (6.3\% vs $3.1 \%$, respectively; Table 7$)$, but this increase was dependent on background insulin use. The incidence of hypoglycaemia events was similar between ipragliflozin and placebo when they were not combined with any other glucose-lowering drugs $(1.3 \%$ vs $0.9 \%$, respectively; Table 7). There was a higher incidence of hypoglycaemia compared with placebo when ipragliflozin was combined with insulin $(32.4 \%$ vs $23.3 \%$, respectively; Supplementary Table S4) or any combination which included insulin (29.7\% vs $14.9 \%$, respectively) compared with non-insulin combinations $(2.6 \%$ vs $2.0 \%$, respectively; Table 7). Overall, hypoglycaemiarelated AEs were mild in severity and no drugrelated hypoglycaemia-related events led to discontinuation. 


\section{DISCUSSION}

This pooled analysis confirmed that ipragliflozin is well tolerated in patients with T2DM, with a safety profile consistent with that observed previously for the SGLT2 inhibitor drug class. The incidence of TEAEs, SAEs and TEAEs leading to discontinuation was comparable between the placebo and ipragliflozin treatment groups, and no new safety signals were identified. TEAEs related to pollakiuria, volume depletion, genital infection and skin and subcutaneous tissue disorders were more common after ipragliflozin treatment compared with placebo, while there were similar rates of UTI, CV events, hypoglycaemia renal disorder, fracture and malignant tumours. TEAEs were generally mild in severity and were managed within standard clinical practice.

For patients treated with ipragliflozin monotherapy, there were no drug-related hypoglycaemia events. The low risk for hypoglycaemia is a well-established advantage of the SGLT2 inhibitor drug class, and is consistent with the results of previous pooled and metaanalyses [33, 47-50]. The hypoglycaemia observed with combination therapy is also consistent with a recent pooled analysis of canagliflozin trials, where hypoglycaemia was reported for $6.9 \%$ patients receiving canagliflozin monotherapy (100 mg) and $44.4 \%$ of patients receiving additional glucose-lowering drugs associated with hypoglycaemia (i.e. insulin, sulfonylurea, glinide) [50]. While a background of sulfonylurea use has been shown previously to increase the risk of hypoglycaemia $[47,48]$, this was not evident in our subgroup analysis, with very low overall rates of hypoglycaemia with added ipragliflozin (3/218, $1.4 \%)$ and placebo $(1 / 96,1.0 \%)$ in patients on sulfonylureas (Supplementary Table S4). Rather, hypoglycaemia in our analysis was almost entirely dependent on background insulin use; rates of drug-related hypoglycaemic events with insulin and non-insulin combinations were $29.1 \%$ and $1.0 \%$ respectively, suggesting that relative overdose of insulin when combined with ipragliflozin was the cause of hypoglycaemia.
The lack of excess UTI and increased incidence of genital infections with ipragliflozin vs placebo are consistent with results from a metaanalysis of 77 randomised controlled trials of SGLT2 inhibitors which showed no significant risk in UTIs vs control $(2526 / 29,086$ vs $1278 / 14,940$; risk ratio [RR] 1.05, 95\% CI 0.98-1.12), but an increased risk of genital infections (1521/24,017 vs 216/12,552; RR 3.30, 95\% CI 2.74-3.99) [51]. A recent safety announcement issued by the US Food and Drug Administration (FDA) warned that cases of Fournier's gangrene, a rare but serious genital infection, have also been reported with SGLT2 inhibitors [38]. In our analysis, no case of Fournier's gangrene was reported, and TEAEs related to genital infection were generally mild and rarely led to treatment discontinuation.

The number of patients with increased urine ketone body levels $(3+)$ was small and no cases of ketoacidosis were reported. Post-marketing surveillance data for ipragliflozin also suggest a low risk for ketoacidosis, with just one case of diabetic ketoacidosis (0.01\%) reported after 24 months, in a large sample of patients in Japan [45]. Although the incidence of ketoacidosis in randomised controlled trials of other SGLT2 inhibitors is also very low [48-50], there is an increased risk compared with placebo $(2.2$ times greater risk in a recent meta-analysis of $\mathrm{CV}$ outcome trials [27]). As a result, drug regulatory agencies have issued warnings about the risk of ketoacidosis with SGLT2 inhibitor use and have provided recommendations to minimise the risk $[52,53]$.

Osmotic diuresis caused by SGLT2 inhibition may potentially lead to volume depletion in susceptible patients [54]. Indeed, the incidence of volume depletion in our analysis was higher for ipragliflozin compared with placebo $(4.9 \%$ vs $1.8 \%$ ). However, these events are mild and have been suggested to reduce over time in a previous study [50]. In a pooled analysis of canagliflozin studies, the majority of volume depletion-related AEs occurred in the first 3 months and then decreased over the course of the next 2 years [50]. In our analysis, the incidence of TEAEs related to renal disorder was similar for ipragliflozin $(0.9 \%)$ and placebo (1.6\%), and the only renal disorder-related TEAE 
observed in the ipragliflozin group that was not observed the placebo group was decreased glomerular filtration rate (GFR; $n=3,0.2 \%$ : Supplementary Table S3). This is consistent with a recent systematic review of SGLT2 clinical trials and regulatory reports that reported a lack of renal safety signal and emphasised that early volume-related reductions in GFR do not cause significant further reduction in renal function [33]. Furthermore, the CREDENCE trial has demonstrated renal protection with canagliflozin in patients with T2DM and chronic kidney disease, over a median follow-up of 2.62 years, despite early reductions in estimated GFR [25]. Evaluation of long-term renal function is limited in the present analysis by the 24-week timeframe.

While ipragliflozin has been reported to improve many cardiometabolic risk factors in patients with T2DM [22], investigation of any cardioprotective effects will require long-term intervention and follow-up. In the EMPA-REG OUTCOME trial [23], the composite endpoint of death from CV causes, non-fatal myocardial infarction and non-fatal stroke decreased by $14 \%(P=0.04)$ in patients treated with empagliflozin after a median follow-up of 3.1 years. $\mathrm{CV}$ benefits have also been reported in longterm outcome studies for canagliflozin [24] and dapagliflozin [26] and in a large, global, realworld study of SGLT2 inhibitors, including ipragliflozin, vs other glucose-lowering drugs [55]. Compared with the patients with established CV diseases in previous outcomes trials, the patient population in our pooled analysis had a relatively low CV risk. While ipragliflozin was associated with fewer CV-related TEAEs than placebo, overall rates were low (one event in ipragliflozin group vs four events in the placebo group). Thus, the low $\mathrm{CV}$ risk and the short timeframe limit any meaningful interpretation of data regarding cardioprotective effects of ipragliflozin in the present analysis.

An FDA Advisory Committee have already raised concerns regarding a potential dapagliflozin-associated risk of bladder and breast cancer [36]. However, meta-analyses of the current evidence from clinical studies of SGLT2 inhibitors $[33,35,56]$ have not identified an overall increased cancer risk, and a recent large- scale dapagliflozin study $(n=17,160)$ has shown statistically lower rates of bladder cancer compared with placebo [26]. Consistent with this, our pooled analysis showed no marked increase in the incidence of any type of cancer. However, given the relatively short-term nature of the studies, further monitoring of the longterm effects of ipragliflozin may be required to continue.

Fracture and lower limb amputation was included as an outcome based on findings from the canagliflozin clinical trials, CANVAS study and CANVAS-R study, which have reported increased incidence of fracture and an approximate two-fold increase in lower limb amputation [24]. However, consistent with the pooled safety findings for empagliflozin and dapagliflozin $[48,49]$ and results of a more recent clinical outcomes study for canagliflozin (CREDENCE study [25]), no increase in fracture was identified with ipragliflozin and no case of lower limb amputation occurred.

Skin and subcutaneous tissue disorders were increased with ipragliflozin treatment in the present study $(7.7 \%$ in ipragliflozin treatment group vs $4.4 \%$ in placebo group), with an incidence approximately $3.3 \%$ higher than placebo. Hypersensitivity AEs with SGLT2 inhibitors have been reported previously in some patients, including a recent report of dermatological AEs in Japan [39]. In a recent pooled analysis of hypersensitivity-related skin AEs for dapagliflozin, the most common skin events were rash (1.1\% with dapagliflozin vs $1.1 \%$ with active or placebo comparator), eczema ( $0.6 \%$ vs $0.8 \%)$, dermatitis $(0.5 \%$ vs $0.4 \%)$ and urticaria $(0.5 \%$ vs $0.2 \%$ ), with few patients discontinuing as a result of hypersensitivity AEs $(\leq 0.2 \%)$ [42]. Consistent with these findings, eczema and skin rash were the most common events reported with ipragliflozin in the present study (Supplementary Table S5). Interim results of an ipragliflozin post-marketing surveillance study in Japan (STELLA-LONG TERM), which included a safety analysis set comprising 11,051 patients, reported 147 cases $(1.3 \%)$ of skin and subcutaneous tissue disorders after 1 year of follow-up [46]. Skin lesions are reported to be well controlled after stopping ipragliflozin and administration of appropriate anti-inflammatory 
drugs. Furthermore, a sub-analysis in the present study revealed that Asian patients with diabetes in both the ipragliflozin and placebo groups had a higher incidence of skin disorder events than non-Asian patients with diabetes (Supplementary Table S5). This differs from the dapagliflozin data, which shows no difference in the incidence of serious hypersensitivity reaction between Asian and non-Asian patients [42]. There have been few studies investigating the potential mechanisms for SGLT2 inhibitorrelated skin lesions and the exact mechanisms remain unknown. One study has reported that ipragliflozin is retained in the skin of rats at a relatively higher concentration compared with other SGLT2 inhibitors [41]. Furthermore, cluster analysis of an in silico 3-D docking simulation indicated a stable ipragliflozin-melanin complex, suggesting a possible role for melanin in ipragliflozin-specific skin and subcutaneous tissue disorders [41]. In any case, accumulation of further clinical case studies will be important for the elucidation of the exact mechanisms.

A major limitation of our pooled safety analysis was the limited patient numbers and short-term duration of the trials, all of which lasted for a maximum of 24 weeks for the placebo comparison. Although the incidence of AEs is often higher at the start of a trial, and would not be affected by the short duration of the trials, some outcomes of special interest, such as CV events and cancer, will require longer-term follow-up data to determine any meaningful results. It should be noted that all trials included patients with T2DM only and findings may not apply to T1DM, for which ipragliflozin is also indicated. Also, while ipragliflozin is approved at $50 \mathrm{mg}$ and $100 \mathrm{mg}$ doses, only data for patients who received $50 \mathrm{mg}$ were included in the pooled analysis, owing to the relatively small patient numbers for the $100 \mathrm{mg}$ dose in comparative studies ( $n=72$ for $100 \mathrm{mg}$ vs $n=1209$ for the $50 \mathrm{mg}$ dose). The summary AE data per dose (Supplementary Table S2) suggest similar incidences between the $50 \mathrm{mg}$ and $100 \mathrm{mg}$ doses for TEAEs (63.8\% and $56.9 \%$, respectively), drug-related TEAEs $(24.7 \%$ vs $25.0 \%)$ and TEAEs leading to discontinuation (3.6\% vs $4.2 \%)$.
Limitations of the pooled methodology include the post hoc nature of the present analyses and outcome definitions that might not be identical across all the studies. Furthermore, the findings from highly selected patient populations in clinical studies do not always reflect real-world outcomes, thus limiting the generalisability of the findings. However, it is noteworthy that the latest findings from ongoing post-marketing surveillance programmes for ipragliflozin support the pooled clinical trial data reported here [44-46].

\section{CONCLUSION}

This pooled analysis of randomised, placebocontrolled trials has confirmed that ipragliflozin is well tolerated in patients with T2DM, with a safety profile consistent with that observed previously for the SGLT2 inhibitor drug class and post-marketing surveillance data. No new safety concerns were identified, confirming that ipragliflozin is well tolerated when used alone or in combination with other glucose-lowering agents.

\section{ACKNOWLEDGEMENTS}

Funding. This study was initiated and supported by Astellas Pharma Inc. The journal's Rapid Service Fee was funded by Astellas Pharma Inc.

Medical Writing Assistance. The authors would like to thank Lisa O'Rourke, PhD, for Cello Health MedErgy for writing support. Writing support was funded by Astellas Pharma Inc.

Authorship. All authors meet the International Committee of Medical Journal Editors (ICMJE) authorship guidelines for this article, take responsibility for the integrity of the work as a whole, and have given their approval for this version to be published. 
Authorship Contributions. YI contributed to study conception, and design. AK contributed to the study design, data acquisition and data analysis. SY contributed to the study design and statistical analysis. All authors interpreted the data and reviewed and edited the manuscript for important intellectual content. All authors have read and approved the final version of the manuscript.

Disclosures. Atsunori Kashiwagi has acted as a consultant for Astellas Pharma Inc., and has received consulting fees/honoraria from Astellas Pharma Inc. He has acted as an expert witness for Sunstar Group Ltd. Yuichiro Ito is an employee of Astellas Pharma Inc. Masahiro Noguchi is an employee of Astellas Pharma Inc. Wim Wilpshaar is an employee of Astellas Pharma Inc. Satoshi Yoshida is an employee of Astellas Pharma Inc. John P. H. Wilding has consulted for Astellas, AstraZeneca, Janssen, Lilly, Mundipharma, Napp, Sanofi, Novo Nordisk and Wilmington Healthcare; received grants from AstraZeneca, Novo Nordisk and Takeda; and personal fees from AstraZeneca, Boehringer Ingelheim, Lilly, Mundipharma, Napp, Sanofi, Novo Nordisk and Takeda. Marina V. Shestakova has nothing to disclose.

Compliance with Ethics Guidelines. This article is based on analysis of data from previously conducted studies. All procedures followed in the original studies involving human participants were in accordance with the ethical standards of the institutional and/or national research committee, and with the 1964 Helsinki declaration and its later amendments or comparable ethical standards. Informed consent was obtained from all individual participants included in the study. An independent ethics committee or institutional review board approved the clinical protocol at each participating centre. All participants provided written informed consent prior to inclusion.

Data Availability. For studies NCT00621868 and NCT01505426, access to anonymized individual participant level data will not be provided as it meets one or more of the exceptions described on http://www.clinicalstudy datarequest.com under "Sponsor Specific Details for Astellas." For all other studies, access to anonymized individual participant level data collected during the study, in addition to supporting clinical documentation, is planned for studies conducted with approved product indications and formulations, as well as compounds terminated during development. Studies conducted with product indications or formulations that remain active in development are assessed after study completion to determine if Individual Participant Data can be shared. Conditions and exceptions are described under the Sponsor Specific Details for Astellas on http://www.clinicalstudydatarequest.com. Study-related supporting documentation is redacted and provided if available, such as the protocol and amendments, statistical analysis plan and clinical study report. Access to participant level data is offered to researchers after publication of the primary manuscript (if applicable) and is available as long as Astellas has legal authority to provide the data. Researchers must submit a proposal to conduct a scientifically relevant analysis of the study data. The research proposal is reviewed by an Independent Research Panel. If the proposal is approved, access to the study data is provided in a secure data sharing environment after receipt of a signed Data Sharing Agreement.

Open Access. This article is distributed under the terms of the Creative Commons Attribution-NonCommercial 4.0 International License (http://creativecommons.org/licenses/ by-nc/4.0/), which permits any noncommercial use, distribution, and reproduction in any medium, provided you give appropriate credit to the original author(s) and the source, provide a link to the Creative Commons license, and indicate if changes were made.

\section{REFERENCES}

1. Gerich JE. Role of the kidney in normal glucose homeostasis and in the hyperglycaemia of diabetes mellitus: therapeutic implications. Diabet Med. 2010;27:136-42. https://doi.org/10.1111/j.1464-54 91.2009.02894.x. 
2. Rahmoune H, Thompson PW, Ward JM, Smith CD, Hong G, Brown J. Glucose transporters in human renal proximal tubular cells isolated from the urine of patients with non-insulin-dependent diabetes. Diabetes. 2005;54:3427-34.

3. Rieg T, Masuda T, Gerasimova M, et al. Increase in SGLT1-mediated transport explains renal glucose reabsorption during genetic and pharmacological SGLT2 inhibition in euglycemia. Am J Physiol Renal Physiol. 2014;306:F188-93. https://doi.org/10.1152 /ajprenal.00518.2013.

4. Mosley JF, Smith L, Everton E, Fellner C, Fellner C. Sodium-glucose linked transporter 2 (SGLT2) inhibitors in the management of type- 2 diabetes: a drug class overview. Pharm Ther. 2015;40:451-62.

5. Fonseca VA, Ferrannini E, Wilding JP, et al. Activeand placebo-controlled dose-finding study to assess the efficacy, safety, and tolerability of multiple doses of ipragliflozin in patients with type 2 diabetes mellitus. J Diabetes Complic. 2013;27:268-73.

6. Kashiwagi A, Kazuta K, Yoshida S, Nagase I. Randomized, placebo-controlled, double-blind glycemic control trial of novel sodium-dependent glucose cotransporter 2 inhibitor ipragliflozin in Japanese patients with type 2 diabetes mellitus. J Diabetes Investig. 2014;5:382-91. https://doi.org/ $10.1111 /$ jdi.12156.

7. Shestakova MV, Wilding JPH, Wilpshaar W, Tretter $\mathrm{R}$, Orlova VL, Verbovoy AF. A phase 3 randomized placebo-controlled trial to assess the efficacy and safety of ipragliflozin as an add-on therapy to metformin in Russian patients with inadequately controlled type 2 diabetes mellitus. Diabetes Res Clin Pract. 2018;146:240-50. https://doi.org/10. 1016/j.diabres.2018.10.018.

8. Kashiwagi A, Takahashi $\mathrm{H}$, Ishikawa $\mathrm{H}$, et al. A randomized, double-blind, placebo-controlled study on long-term efficacy and safety of ipragliflozin treatment in patients with type 2 diabetes mellitus and renal impairment: results of the LongTerm ASP1941 Safety Evaluation in Patients with Type 2 Diabetes. Diabetes Obes Metab. 2015;17: 152-60. https://doi.org/10.1111/dom.12403.

9. Kashiwagi A, Kazuta K, Takinami Y, Yoshida S, Utsuno A, Nagase I. Ipragliflozin improves glycemic control in Japanese patients with type 2 diabetes mellitus: the BRIGHTEN study. Diabetol Int. 2015;6:8-18. https://doi.org/10.1007/s13340-0140164-0.

10. Wilding JPH, Ferrannini E, Fonseca VA, Wilpshaar W, Dhanjal P, Houzer A. Efficacy and safety of ipragliflozin in patients with type 2 diabetes inadequately controlled on metformin: a dose-finding study. Diabetes Obes Metab. 2013;15:403-9.
11. Kashiwagi A, Kazuta K, Goto K, Yoshida S, Ueyama E, Utsuno A. Ipragliflozin in combination with metformin for the treatment of Japanese patients with type 2 diabetes: ILLUMINATE, a randomized, double-blind, placebo-controlled study. Diabetes Obes Metab. 2015;17:304-8.

12. Kashiwagi A, Akiyama N, Shiga T, et al. Efficacy and safety of ipragliflozin as an add-on to a sulfonylurea in Japanese patients with inadequately controlled type 2 diabetes: results of the randomized, placebocontrolled, double-blind, phase III EMIT study. Diabetol Int. 2015;6:125-38.

13. Kashiwagi A, Shiga T, Akiyama N, et al. Efficacy and safety of ipragliflozin as an add-on to pioglitazone in Japanese patients with inadequately controlled type 2 diabetes: a randomized, double-blind, placebo-controlled study (the SPOTLIGHT study). Diabetol Int. 2015;6:104-16.

14. Ishihara H, Yamaguchi S, Nakao I, Asahina S, Sakatani T. Efficacy and safety of ipragliflozin as add-on therapy to insulin in Japanese patients with type 2 diabetes mellitus (IOLITE): a 36-week, openlabel extension of a 16-week, randomized, placebocontrolled, double-blind study. Diabetol Int. 2018;2018:1-14.

15. Lu C-H, Min KW, Chuang L-M, Kokubo S, Yoshida S, Cha B-S. Efficacy, safety, and tolerability of ipragliflozin in Asian patients with type 2 diabetes mellitus and inadequate glycemic control with metformin: results of a phase 3 randomized, placebo-controlled, double-blind, multicenter trial. J Diabetes Investig. 2016;7:366-73. https://doi.org/ 10.1111/jdi.12422.

16. Han K-A, Chon S, Chung CH, et al. Efficacy and safety of ipragliflozin as an add-on therapy to sitagliptin and metformin in Korean patients with inadequately controlled type 2 diabetes mellitus: a randomized controlled trial. Diabetes Obes Metab. 2018;20:2408-15. https://doi.org/10.1111/dom.13 394.

17. Storgaard H, Gluud LL, Bennett C, et al. Benefits and harms of sodium-glucose co-transporter 2 inhibitors in patients with type 2 diabetes: a systematic review and meta-analysis. PLoS One. 2016;11:e0166125. https://doi.org/10.1371/journal. pone.0166125.

18. Kashiwagi A, Yoshida S, Kawamuki K, et al. Effects of ipragliflozin, a selective sodium-glucose cotransporter 2 inhibitor, on blood pressure in Japanese patients with type 2 diabetes mellitus: a pooled analysis of six randomized, placebo-controlled clinical trials. Diabetol Int. 2017;8:76-86. https:// doi.org/10.1007/s13340-016-0283-X. 
19. Min K-W, Ku BJ, Lee J-H, et al. Addition of ipragliflozin to metformin treatment in Korean patients with type 2 diabetes mellitus: subgroup analysis of a phase 3 trial. Diabetes Metab J. 2017;41:135. https://doi.org/10.4093/dmj.2017.41.2.135.

20. Oliva RV, Bakris GL. Blood pressure effects of sodium-glucose co-transport 2 (SGLT2) inhibitors. J Am Soc Hypertens. 2014;8:330-9. https://doi.org/ 10.1016/j.jash.2014.02.003.

21. Tikkanen I, Narko K, Zeller C, et al. Empagliflozin reduces blood pressure in patients with type 2 diabetes and hypertension. Diabetes Care. 2015;38:420-8. https://doi.org/10.2337/dc14-1096.

22. Kashiwagi A, Sakatani T, Nakamura I, et al. Improved cardiometabolic risk factors in Japanese patients with type 2 diabetes treated with ipragliflozin: a pooled analysis of six randomized, placebocontrolled trials. Endocr J. 2018;65:693-705. https://doi.org/10.1507/endocrj.EJ17-0491.

23. Zinman B, Wanner C, Lachin JM, et al. Empagliflozin, cardiovascular outcomes, and mortality in type 2 diabetes. N Engl J Med. 2015;373:2117-28. https://doi.org/10.1056/NEJMoa1504720.

24. Neal B, Perkovic V, Mahaffey KW, et al. Canagliflozin and cardiovascular and renal events in type 2 diabetes. N Engl J Med. 2017;377:644-57. https:// doi.org/10.1056/NEJMoa1611925.

25. Perkovic V, Jardine MJ, Neal B, et al. Canagliflozin and renal outcomes in type 2 diabetes and nephropathy. N Engl J Med. 2019;2019:NEJMoa1811744. https:// doi.org/10.1056/nejmoa1811744.

26. Wiviott SD, Raz I, Bonaca MP, et al. Dapagliflozin and cardiovascular outcomes in type 2 diabetes. N Engl J Med. 2019;380:347-57. https://doi.org/10. 1056/NEJMoa1812389.

27. Zelniker TA, Wiviott SD, Raz I, et al. SGLT2 inhibitors for primary and secondary prevention of cardiovascular and renal outcomes in type 2 diabetes: a systematic review and meta-analysis of cardiovascular outcome trials. Lancet. 2019;393:31-9. https://doi. org/10.1016/S0140-6736(18)32590-X.

28. Kaku K, Isaka H, Toyoshima J, Sakatani T. Clinical pharmacology study of ipragliflozin in Japanese patients with type 1 diabetes mellitus: a phase 2, randomized, placebo-controlled trial. Diabetes Obes Metab. 2019;21:1445-54. https://doi.org/10. 1111/dom.13679.

29. Dandona P, Mathieu C, Phillip M, et al. Efficacy and safety of dapagliflozin in patients with inadequately controlled type 1 diabetes (DEPICT-1): 24 week results from a multicentre, double-blind, phase 3 , randomised controlled trial. Lancet Diabetes
Endocrinol. 2017;5:864-76. https://doi.org/10. 1016/S2213-8587(17)30308-X.

30. Rosenstock J, Marquard J, Laffel LM, et al. Empagliflozin as adjunctive to insulin therapy in type 1 diabetes: the EASE trials. Diabetes Care. 2018;41: 2560-9. https://doi.org/10.2337/dc18-1749.

31. Kaku K, Isaka H, Sakatani T, Toyoshima J. Efficacy and safety of ipragliflozin add-on therapy to insulin in Japanese patients with type 1 diabetes mellitus: a randomized, double-blind, phase 3 trial. Diabetes Obes Metab. 2019;2019:dom.13807. https://doi. org/10.1111/dom.13807.

32. Filippas-Ntekouan S, Filippatos TD, Elisaf MS. SGLT2 inhibitors: are they safe? Postgrad Med. 2018;130:72-82. https://doi.org/10.1080/00325481. 2018.1394152.

33. Rådholm K, Wu JH, Wong MG, et al. Effects of sodium-glucose cotransporter- 2 inhibitors on cardiovascular disease, death and safety outcomes in type 2 diabetes-a systematic review. Diabetes Res Clin Pract. 2018;140:118-28. https://doi.org/10. 1016/j.diabres.2018.03.027.

34. Fadini GP, Bonora BM, Avogaro A. SGLT2 inhibitors and diabetic ketoacidosis: data from the FDA Adverse Event Reporting System. Diabetologia. 2017;60:1385-9. https://doi.org/10.1007/s00125017-4301-8.

35. Tang H, Dai Q, Shi W, Zhai S, Song Y, Han J. SGLT2 inhibitors and risk of cancer in type 2 diabetes: a systematic review and meta-analysis of randomised controlled trials. Diabetologia. 2017;60:1862-72. https://doi.org/10.1007/s00125-017-4370-8.

36. Food and Drug Administration. FDA briefing document, NDA 202293 Dapagliflozin tablets, 5 and $10 \mathrm{mg}$. Advisory Committee Meeting. 2013.

37. Watts NB, Bilezikian JP, Usiskin K, et al. Effects of canagliflozin on fracture risk in patients with type 2 diabetes mellitus. J Clin Endocrinol Metab. 2016;101:157-66. https://doi.org/10.1210/jc.20153167.

38. Food and Drug Administration. FDA warns about rare occurrences of a serious infection of the genital area with SGLT2 inhibitors for diabetes. Safety Announcement 2018. https://www.fda.gov/Drugs/ DrugSafety/ucm617360.htm. Accessed 14 Jan 2019.

39. Yabe D, Nishikino R, Kaneko M, Iwasaki M, Seino Y. Short-term impacts of sodium/glucose co-transporter 2 inhibitors in Japanese clinical practice: considerations for their appropriate use to avoid serious adverse events. Expert Opin Drug Saf. 2015;14:795-800. https://doi.org/10.1517/1474033 8.2015.1034105. 
40. Vasapollo P, Cione E, Luciani F, Gallelli L. Generalized intense pruritus during canagliflozin treatment: is it an adverse drug reaction? Curr Drug Saf. 2018;13:38-40. https://doi.org/10.2174/15748863 11666160405110515.

41. Sakaeda T, Kobuchi S, Yoshioka R, et al. Susceptibility to serious skin and subcutaneous tissue disorders and skin tissue distribution of sodiumdependent glucose co-transporter type 2 (SGLT2) inhibitors. Int J Med Sci. 2018;15:937-43. https:// doi.org/10.7150/ijms.22224.

42. Mellander A, Billger M, Johnsson E, Träff AK, Yoshida S, Johnsson K. Hypersensitivity events, including potentially hypersensitivity-related skin events, with dapagliflozin in patients with type 2 diabetes mellitus: a pooled analysis. Clin Drug Investig. 2016;36:925-33. https://doi.org/10.1007/ s40261-016-0438-3.

43. Poole RM, Dungo RT. Ipragliflozin: first global approval. Drugs. 2014;74:611-7. https://doi.org/10. 1007/s40265-014-0204-X.

44. Yokote K, Terauchi Y, Nakamura I, Sugamori H. Real-world evidence for the safety of ipragliflozin in elderly Japanese patients with type 2 diabetes mellitus (STELLA-ELDER): final results of a post-marketing surveillance study. Expert Opin Pharmacother. 2016;17:1995-2003. https://doi.org/ 10.1080/14656566.2016.1219341.

45. Nakamura I, Maegawa H, Tobe K, Tabuchi H, Uno S. Safety and efficacy of ipragliflozin in Japanese patients with type 2 diabetes in real-world clinical practice: interim results of the STELLA-LONG TERM post-marketing surveillance study. Expert Opin Pharmacother. 2018;19:189-201. https://doi.org/ 10.1080/14656566.2017.1408792.

46. Nakamura I, Maegawa H, Tobe K, Uno S. Safety and effectiveness of ipragliflozin for type 2 diabetes in japan: 12-month interim results of the STELLALONG TERM post-marketing surveillance study. Adv Ther. 2019. https://doi.org/10.1007/s12325019-0895-1.

47. Vasilakou D, Karagiannis T, Athanasiadou E, et al. Sodium-glucose cotransporter 2 inhibitors for type 2 diabetes. Ann Intern Med. 2013;159:262. https:// doi.org/10.7326/0003-4819-159-4-20130820000007.

48. Kohler S, Zeller C, Iliev H, Kaspers S. Safety and tolerability of empagliflozin in patients with type 2 diabetes: pooled analysis of phase I-III clinical trials. Adv Ther. 2017;34:1707-26. https://doi.org/10. 1007/s12325-017-0573-0.

49. Jabbour S, Seufert J, Scheen A, Bailey CJ, Karup C, Langkilde AM. Dapagliflozin in patients with type 2 diabetes mellitus: a pooled analysis of safety data from phase IIb/III clinical trials. Diabetes Obes Metab. 2018;20:620-8. https://doi.org/10.1111/ dom.13124.

50. Qiu R, Balis D, Xie J, Davies MJ, Desai M, Meininger G. Longer-term safety and tolerability of canagliflozin in patients with type 2 diabetes: a pooled analysis. Curr Med Res Opin. 2017;33:553-62. https://doi.org/10.1080/03007995.2016.1271780.

51. Liu J, Li L, Li S, et al. Effects of SGLT2 inhibitors on UTIs and genital infections in type 2 diabetes mellitus: a systematic review and meta-analysis. Sci Rep. 2017;7:2824. https://doi.org/10.1038/s41598017-02733-w.

52. Food and Drug Administration. FDA Drug Safety Communication: FDA warns that SGLT2 inhibitors for diabetes may result in a serious condition of too much acid in the blood. 2015. https://www.fda. gov/downloads/Drugs/DrugSafety/UCM446954.\% OApdf. Accessed 14 Jan 2019.

53. European Medicines Agency. SGLT2 Inhibitors. EMA confirms recommendations to minimise ketoacidosis risk with SGLT2 inhibitors for diabetes 2016. https://www.ema.europa.eu/en/medicines/ human/referrals/sglt2-inhibitors. Accessed 14 Jan 142019.

54. Reed JW. Impact of sodium-glucose cotransporter 2 inhibitors on blood pressure. Vasc Health Risk Manag. 2016;12:393-405. https://doi.org/10.2147/ VHRM.S111991.

55. Kosiborod M, Lam CSP, Kohsaka S, et al. Cardiovascular events associated with SGLT-2 inhibitors versus other glucose-lowering drugs: the CVD-REAL 2 study. J Am Coll Cardiol. 2018;71:2628-39. https://doi.org/10.1016/j.jacc.2018.03.009.

56. Wu JHY, Foote C, Blomster J, et al. Effects of sodium-glucose cotransporter- 2 inhibitors on cardiovascular events, death, and major safety outcomes in adults with type 2 diabetes: a systematic review and meta-analysis. Lancet Diabetes Endocrinol. 2016;4:411-9. https://doi.org/10.1016/ S2213-8587(16)00052-8. 